\title{
Extracellular vesicles derived from human placental mesenchymal stem cells alleviate experimental colitis in mice by inhibiting inflammation and oxidative stress
}

\author{
LIYUN DUAN $^{1-3}$, HAOYAN HUANG ${ }^{2}$, XIAOTONG ZHAO $^{4}$, MANQIAN ZHOU $^{5}$, SHANG CHEN $^{2}$, \\ CHEN WANG $^{2}$, ZHIBO HAN ${ }^{6-8}$, ZHONG-CHAO HAN $^{6-8}$, ZHIKUN GUO $^{4}$, ZONGJIN LI $^{2-4}$ and XIAOCANG CAO ${ }^{1}$ \\ ${ }^{1}$ Department of Hepato-Gastroenterology, Tianjin Medical University General Hospital, Tianjin Medical University; \\ ${ }^{2}$ School of Medicine, Nankai University; ${ }^{3}$ The Key Laboratory of Bioactive Materials, Ministry of Education, \\ College of Life Sciences, Nankai University, Tianjin 300071; ${ }^{4}$ Henan Key Laboratory of Medical Tissue Regeneration, \\ Xinxiang Medical University, Xinxiang, Henan 453003 ; ${ }^{5}$ Department of Radiation Oncology, Tianjin Union Medical Center, \\ Tianjin 300121; ${ }^{6}$ Beijing Engineering Laboratory of Perinatal Stem Cells, Beijing Institute of Health and Stem Cells, \\ Health and Biotech Co., Beijing 100176; ${ }^{7}$ Tianjin Key Laboratory of Engineering Technologies for Cell Pharmaceutical, \\ National Engineering Research Center of Cell Products, AmCellGene Co., Ltd., Tianjin 300457; \\ ${ }^{8}$ Jiangxi Engineering Research Center for Stem Cell, Shangrao, Jiangxi 334001, P.R. China
}

Received February 8, 2020; Accepted July 2, 2020

DOI: $10.3892 /$ ijmm.2020.4679

\begin{abstract}
Mesenchymal stem cells (MSCs) are pluripotent cells that can be applied to the treatment of immune disorders, including inflammatory bowel disease (IBD). The therapeutic effects of MSCs have been mostly attributed to the secretion of soluble factors with paracrine actions, such as extracellular vesicles (EVs), which may play a relevant role in the repair of damaged tissues. In the present study, a mouse model of colitis was induced with the use of trinitrobenzene sulfonic acid (TNBS). EVs derived from human placental mesenchymal stem cells (hP-MSCs) were used for the treatment of colitis by in situ injection. Clinical scores were applied to verify the therapeutic effects of EVs on mice with colitis. Inflammation in the colon was evaluated by measuring the levels of various inflammatory cytokines. The content of reactive oxygen species (ROS) was detected by the use of molecular imaging methods for real-time tracking and the therapeutic effects of EVs on mucosal healing in mice with colitis were evaluated. The results revealed that the injection of EVs regulated the balance of pro-inflammatory and anti-inflammatory cytokines
\end{abstract}

Correspondence to: Professor Zongjin Li, School of Medicine, Nankai University, 94 Weijin Road, Tianjin 300071, P.R. China E-mail: zongjinli@nankai.edu.cn

Professor Xiaocang Cao, Department of Hepato-Gastroenterology, Tianjin Medical University General Hospital, Tianjin Medical University, 154 Anshan Road, Tianjin 300071, P.R. China

E-mail: docxccao@163.com

Key words: mesenchymal stem cells, extracellular vesicles, inflammatory bowel disease, inflammation, oxidative stress in colon tissue. Treatment with EVs also suppressed oxidative stress by decreasing the activity of myeloperoxidase (MPO) and ROS. Histological analysis further confirmed that the EVs significantly promoted mucosal healing, as reflected by the promotion of the proliferation of colonic epithelial cells and the maintenance of tight junctions. Taken together, the findings of the present study demonstrated that EVs derived from hP-MSCs alleviated TNBS-induced colitis by inhibiting inflammation and oxidative stress. These findings may provide a novel theoretical basis for the EV-based treatment of IBD.

\section{Introduction}

Inflammatory bowel disease (IBD), including ulcerative colitis (UC) and Crohn's disease (CD), are chronic relapsing intestinal pathologies triggered by undefined etiological factors $(1,2)$. The pathogenesis of IBD is complex, and no consensus theory is yet available to fully elucidate the development of IBD. The development and progression of IBD may involve a number of factors, such as the environment, genetic and intestinal infections, immunity dysregulation and mucosal barrier defects (3-5). Currently, the therapies for IBD focus on controlling active inflammatory reactions and regulating intestinal immune disorders with the use of immunosuppressive agents for example, which are considered the most effective drugs for the treatment of IBD; however, these have significant side-effects (6).

Previous studies have demonstrated that mesenchymal stem cells (MSCs) exert therapeutic effects against several immune disorders, including IBD due to their potent immunomodulatory effects and tissue regenerative potential (7-9). The paracrine theory of stem cell action has provided new insight into the mechanisms of MSCs in the treatment of several diseases $(10,11)$. Increasing evidence has indicated that MSCs 
regulate the repair of damaged tissues and immune balance through their paracrine soluble factors (12-15). Extracellular vesicles (EVs), tiny membrane vesicles that can be secreted by the majority of cells, have a lipid bilayer membrane structure of approximately 30-200 nm in diameter (12). Recent studies have found that EVs released by cells, acting as mediators of MSC paracrine actions, can be used to communicate between cells (16-18). MSC-derived EVs (MSC-EVs) contain a wide variety of bioactive substances, such as mRNAs, microRNAs (miRNAs or miRs) and proteins, which are not easily degraded (18-20). Furthermore, several studies have indicated that MSC-EVs exert therapeutic effects similar to those of MSCs $(11,12,21,22)$. It has recently been found that the paracrine function of MSCs is mediated by EVs at least to a certain extent. EVs are mainly released from the endosomal compartment and contain certain bioactive substances, such as mRNAs, miRNAs and proteins from their cells of origin (23). Researchers have further confirmed the endosomal origin of MSC-EVs by using the method of lipid raft composition detection (24). MSCs can promote the healing of tissue ischemic diseases by secreting proangiogenic secretory proteins (10). It has recently been further demonstrated that MSC-derived EVs act as paracrine effectors of angiogenesis; however, which components of the EVs proteome are responsible for the identification of this role remains unknown. A previous study performed proteomics analysis and demonstrated that MSC-derived EVs included angiogenic paracrine effectors, which have the potential to treat tissue ischemic diseases (25). Compared with stem cell therapy, EVs do not cause acute immune rejection, do not constitute a risk of tumor formation, and can be easily stored and transported (13). Furthermore, it has been demonstrated that the administration of EVs derived from umbilical cord-MSCs can alleviate colitis in mice, and EVs are administered via tail vein injection to mice (26). Thus, MSC-EVs are likely to become a novel and more efficient cell-free therapy approach for IBD.

In the present study, it was hypothesized that the in situ injection of human placental (hP-)MSC-derived EVs would significantly improve the clinical symptoms and exert beneficial effects by promoting mucosal healing via the inhibition of inflammation and oxidative stress in mice with colitis. It was also hypothesized that the method of administration of the EVs would ensure that they localized directly and rapidly in the damaged intestine for a longer period of time in order to enhance their therapeutic effects. To examine this hypothesis, the therapeutic effects of EVs were assessed in a murine model of colitis induced by trinitrobenzene sulfonic acid (TNBS). Inflammation, oxidative stress and mucosal healing were also evaluated. The findings suggest that EVs derived from hP-MSCs may represent a novel therapeutic approach for IBD by in situ injection.

\section{Materials and methods}

Cells and cell culture. The hP-MSCs used in the present study were provided by Cell Products of National Engineering Research Center/Tianjin Amcellgene Engineering Co., Ltd. The hP-MSCs were cultured in Dulbecco's modified Eagle's medium (DMEM)/F12 medium (Gibco; Thermo Fisher Scientific, Inc.) with $10 \%$ bovine EV-free fetal bovine serum (FBS; HyClone; Cytiva), 1\% L-glutamine (Gibco; Thermo Fisher Scientific, Inc.), 1\% non-essential amino acid (NEAA) and 1\% $100 \mathrm{U} / \mathrm{ml}$ penicillin/streptomycin (Gibco; Thermo Fisher Scientific, Inc.). Bovine EV-free FBS was obtained by ultracentrifugation at $1,00,000 \mathrm{x} \mathrm{g}$ for $70 \mathrm{~min}$ at $4^{\circ} \mathrm{C}$, and filtered using a $100 \mathrm{~nm}$ filter (27).

Isolation and characterization of EVs. EVs were purified from supernatants of hP-MSCs by differential centrifugation $(12,13,15)$. In brief, hP-MSCs were cultured in medium containing $10 \% \mathrm{EV}$-free FBS for $48 \mathrm{~h}$, and the supernatant was then collected. The supernatant was then subjected to sequential centrifugation steps at $500 \mathrm{x} g$ for $10 \mathrm{~min}$ to remove any cell contaminations, at $2,000 \mathrm{x} \mathrm{g}$ for $20 \mathrm{~min}$ to remove apoptotic bodies, and at 5,000 x g for $30 \mathrm{~min}$ to remove cell debris at $4^{\circ} \mathrm{C}$. The resulting supernatant was then filtered using $0.2 \mu \mathrm{m}$ filters (Merck KGaA). The EVs were then harvested by centrifugation at $130,000 \mathrm{x} \mathrm{g}$ for $2 \mathrm{~h}$ in a SW32 Ti rotor (L-100XP Ultracentrifuge, Beckman Coulter) at $4^{\circ} \mathrm{C}$. Finally, the EV pellets were resuspended in PBS and ultracentrifuged again at $130,000 \times \mathrm{g}$ for $2 \mathrm{~h}$ to discard the contaminating proteins, and the purified EVs were harvested. The morphology of the EVs was examined by transmission electron microscopy (TEM; Talos F200C, Thermo Fisher Scientific, Inc.). The particle size of the EV pellets was measured by dynamic light scattering using a BI-200SM laser scattering instrument (ZetaPALS, Brookhaven Instruments) at $20^{\circ} \mathrm{C}$. The protein concentrations of the EVs were measured using a BCA Protein assay kit (Promega Corporation) according to the manufacturer's instructions. The presence of known EV markers, including CD63 (1:1,000 dilution, ab216130, Abcam), CD9 (1:1,000 dilution, ab92726, Abcam), ALIX (1:1,000 dilution, ab186429, Abcam) and GM130 (1:1,000 dilution, ab52649, Abcam) were examined by western blot analysis.

Western blot analysis. Mouse colon tissues (described below) used for western blot analysis were lysed on ice in radioimmunoprecipitation assay (RIPA) buffer (Beijing Solarbio Science $\&$ Technology Co., Ltd.), and its quantification was measured using the $\mathrm{BCA}^{\mathrm{TM}}$ Protein assay kit (Pierce; Thermo Fisher Scientific, Inc.) according to the manufacturer's instructions. The equal amount proteins $(30 \mu \mathrm{g})$ were separated by $12 \%$ sodium dodecyl sulfate polyacrylamide gel electrophoresis (SDS-PAGE; Invitrogen; Thermo Fisher Scientific, Inc.) and transferred to polyvinylidene difluoride membranes (PVDF; EMD Millipore). The membranes were blocked with 5\% non-fat milk in tri-sec-buffered saline/Tween-20 (TBST) buffer (20 mM Tris- $\mathrm{HCl}, \mathrm{pH} 7.6,136 \mathrm{mM} \mathrm{NaCl}$ and $0.1 \%$ Tween-20). After blocking with 5\% non-fat milk for $2 \mathrm{~h}$, the membranes were incubated with primary antibodies overnight at $4^{\circ} \mathrm{C}$, and then for $2 \mathrm{~h}$ at room temperature with horseradish peroxidase-conjugated goat anti-rabbit secondary antibodies (1:5,000 dilution, ab97051, Abcam). The Pierce enhanced chemiluminescence western blotting substrate (EMD Millipore) was used to detect the signal. The primary antibodies used for western blot analysis were as follows: Rabbit anti-CD9 (1:1,000 dilution, ab92726, Abcam), CD63 (1:1,000 dilution, ab216130, Abcam), GM130 (1:1,000 dilution, ab52649, Abcam), and rabbit anti-interleukin (IL)-1 $\beta$ (1:1,000 dilution, WL02257, Wanleibio), IL-6 (1:1,000 dilution, ab229381, 
Abcam), tumor necrosis factor (TNF)- $\alpha$ (1:1,000 dilution, WL01581, Wanleibio), IL-10 (1:1,000 dilution, ab271261, Abcam), interferon (IFN)- $\gamma$ (1:1,000 dilution, WL02440, Wanleibio), EpCAM (1:1,000 dilution, WL01375, Wanleibio). The above-mentioned antibodies were used following the manufacturer's instructions. Clarity Western ECL substrate (Bio-Rad Laboratories, Inc.) and ChemiDoc ${ }^{\mathrm{TM}}$ MP Imaging System (Bio-Rad Laboratories, Inc.) were applied to detect the blots and for visualization. In addition, ImageJ version 1.46 (Rawak Software, Inc.) was used to quantify the blots.

Animal model. For all the experiments, 8-week-old male $\mathrm{BALB} / \mathrm{c}$ mice were utilized that were purchased from the Laboratory Animal Center of the Academy of Military Medical Science. The mice were housed in a standard animal laboratory where the temperature was maintained at $25^{\circ} \mathrm{C}$ with a humidity level of $30-60 \%$. The animals were provided with free access to food and water. The protocols involved animals and the experimental procedures of the present study were approved by the Nankai University Animal Care and Use Committee guidelines (approval no. 20170022) and conducted according to the international regulations of the usage and welfare of laboratory animals. All experimental procedures were conducted in accordance with the Tianjin Committee for the Use and Care of Laboratory Animals. The male BALB/c mice were randomly divided into 3 groups ( $n=8 /$ group) as follows: The sham-operated group (Sham group), the IBD group (PBS group) and the EV-treated IBD group (EV group). As mentioned above, colitis was induced in the mice with TNBS (Sigma-Aldrich; Merck KGaA) (28). The TNBS-induced model of colitis can be established by a single enema without prior sensitization and with a long duration of inflammation, and it is a dynamic process of transformation from acute inflammation to chronic inflammation. The advantages of the TNBS-induced model of colitis include the reproducibility and the technical simplicity. In brief, TNBS $(100 \mathrm{mg} / \mathrm{kg})$ dissolved in $50 \%$ ethanol was slowly administered into the colon via a catheter equipped approximately $3.5 \mathrm{~cm}$ into the anus. The mouse was suspended for $1 \mathrm{~min}$ to allow the drug to fully absorb. On the first day after modeling, the mice were anesthetized by $4 \%$ chloral hydrate $(350 \mathrm{mg} / \mathrm{kg})$ and a laparotomy was performed. A total of $200 \mu \mathrm{g}$ EVs suspended in PBS were injected into the mice in the EV group in situ by injection at a $60 \mu \mathrm{l}$ total volume. The position of in situ administration were the injury colon mesangial margin. The same volume of PBS was administered to the mice in the PBS group as the control. The sham-operated mice were subjected to the same surgical procedure without colitis or EV injection. The body weight, stool consistency and mental state of the mice were measured daily. The mice were monitored every $12 \mathrm{~h}$ within a period of 1 week to examine their health and behavior. The mice were then euthanatized by cervical dislocation when the following humane endpoints were reached: The mice lost weight rapidly, and the loss of body weight were $15-20 \%$ of their original weight; exhibited a complete loss of appetite for up to $24 \mathrm{~h}$; were unable to eat and drink by themselves; exhibited severe persistent gastrointestinal symptoms (persistent diarrhea, intestinal obstruction, intussusception and peritonitis). Respiratory arrest, no heartbeat and no blink reflex were used to confirm mouse death. No mice died before meeting these endpoints. The animal experiment strictly adhered to the principles of using the least number of animals to complete the experiment and minimizing the pain of the experimental animals. All mice were euthanized by cervical dislocation at 3 days after EVs treatment (apart from those included in the survival analysis), and the colon tissues were then collected for subsequent analysis. For in vivo EV trafficking assay, $200 \mu \mathrm{g}$ EVs were incubated with $1 \mu \mathrm{mol} / 1 \mathrm{Dil}$ (Beijing Ouhe Technology Co., Ltd.). At $30 \mathrm{~min}$ after incubation, the labeled EVs were collected and injected into the mice. The presence of labeled EVs in colon tissues was detected in a live animal imaging system.

Histological analysis. At day 3 following TNBS enema, the mice were euthanized, and colonic segments were harvested. In order to determine the injury to the colon mucosa, the paraffin-embedded sections were stained with hematoxylin and eosin (H\&E) (Beyotimebio) according to standardized procedures. Colonic segments were washed in PBS, fixed in $4 \%$ paraformaldehyde ( $\mathrm{pH} 7.4$ ), embedded in paraffin, and stained with H\&E. The colon sections were stained using hematoxylin for 5-10 min, rinsed with distilled water for $1 \mathrm{~min}$, separated color with $0.5 \%$ alcohol hydrochloric acid at $37^{\circ} \mathrm{C}$. The colon sections were then stained with eosin for 2-5 min, dehydrated using graded ethanol, vitrification by dimethylbenzene, and mounted with neutral balsam at $37^{\circ} \mathrm{C}$. Immunohistochemical staining was carried out to determine the inflammation, and proliferation and apoptosis of the damaged intestinal tissue from the mice in the different groups. The paraffin-embedded sections were dewaxed by xylene and hydrated in a graded series of ethanol. The sections were treated with the appropriate amount of endogenous peroxidase blocker and incubate for $10 \mathrm{~min}$ at room temperature $\left(37^{\circ} \mathrm{C}\right)$ to inactivate endogenous peroxidase activity. The sections were then incubated with primary antibodies overnight at $4^{\circ} \mathrm{C}$ for myeloperoxidase (MPO) (1:150 dilution, WL02355, Wanleibio), Ki67 (1:100 dilution, WL01384a, Wanleibio) and EpCAM (1:1,000 dilution, WL01375, Wanleibio). The sections were then incubated with horseradish peroxidase-conjugated goat anti-rabbit secondary antibody (1:5,000 dilution, ab97051, Abcam) for $20 \mathrm{~min}$ at $37^{\circ} \mathrm{C}$, and DAB was used as the substrate. Immunohistochemical positive staining was brown. The sections obtained were examined with an optical microscope (Olympus BX51). For immunofluorescence staining, the colon samples were embedded into optimal cutting temperature (OCT) compound (Sakura Finetek). Samples were cut into 6- $\mu \mathrm{m}$-thick sections. The sections were then incubated with primary antibodies overnight at $4^{\circ} \mathrm{C}$ for EpCAM (1:200 dilution, ab71916, Abcam). The sections were incubated with fluorescently labeled secondary antibodies (Alexa Fluor 488 goat antimouse, 1:1,000 dilution, A-21151, Thermo Fisher Scientific, Inc.) for $2 \mathrm{~h}$ at $37^{\circ} \mathrm{C}$, and the sections were then counterstained with DAPI (Abcam) for $5-10 \mathrm{~min}$ at $37^{\circ} \mathrm{C}$ to identify cell nuclei (Olympus BX51). Images were analyzed using ImageJ version 1.46 (Rawak Software, Inc.).

Bioluminescence imaging. To examine the severity of injury to the intestine, reactive oxygen species (ROS) were detected in the mice for 1 week via an intraperitoneal injection of luminol $(10 \mathrm{mg} / \mathrm{kg}$; Sigma-Aldrich; Merck KGaA). The luminol stock 
A

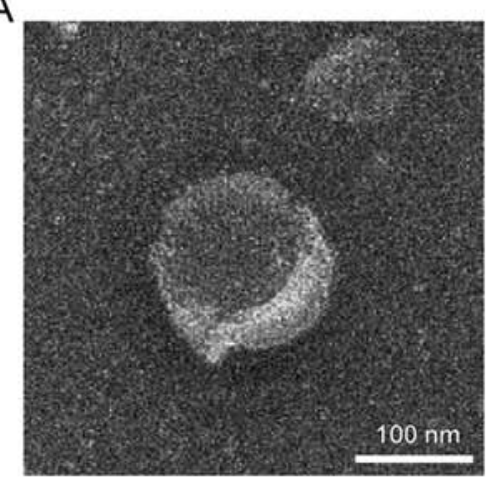

EVs

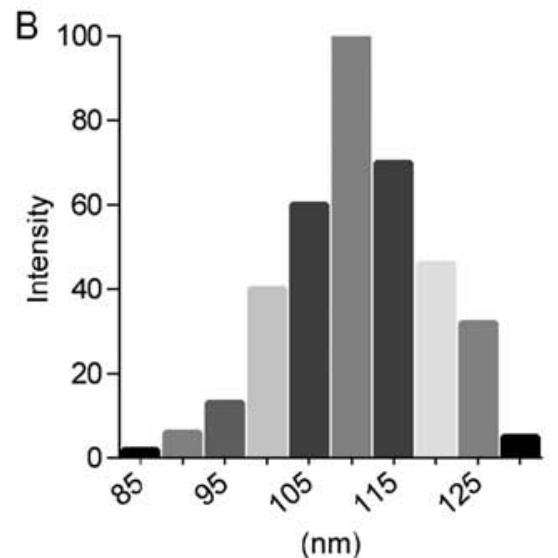

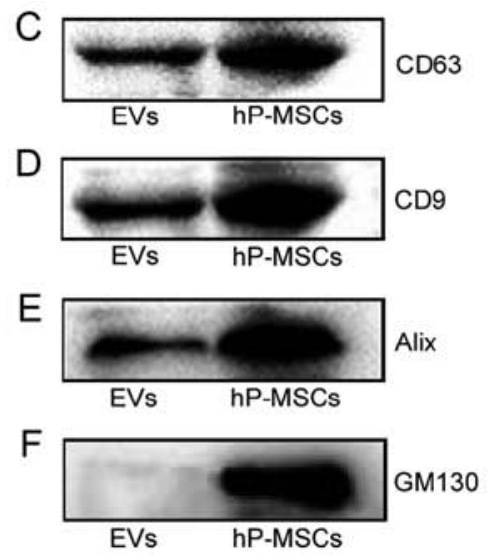

Figure 1. Characteristics of EVs. (A) TEM image of EVs. Scale bar, $100 \mathrm{~nm}$. (B) Size distribution measure of EVs by dynamic light scattering. (C-F) Western blot analysis of CD9, CD63, Alix, GM130 in hP-MSCs and EVs. EVs, extracellular vesicles; hP-MSCs, human placenta-derived mesenchymal stem cells.

solutions were prepared in normal saline prior to injection. The mice were imaged with the IVIS Lumina II system (Xenogen Corp.) 5 min after the injection of the substrate. Bioluminescence signals were quantified in units of maximum photons per second per centimeter squared per steradian (photons $/ \mathrm{sec} / \mathrm{cm}^{2} / \mathrm{sr}$ ) as previously described (29-31). All mice were euthanized by cervical dislocation at 7 days after the experiment.

$R N A$ isolation and reverse transcription-quantitative PCR $(R T-q P C R)$. The extracted colon tissue were suspended in TRIzol reagent (Invitrogen; Thermo Fisher Scientific, Inc.) and total RNA was extracted from the colon samples according to the manufacturer's instructions. The BioScript All-in-One cDNA Synthesis SuperMix (Bimake) was used to produce cDNA by reverse transcription. Gene expression was analyzed by ABI 7500 Fast Real-Time PCR System (Bio-Rad Laboratories, Inc.) using a FastStart Universal SYBR-Green Master (Roche Diagnostics) according to the manufacturer's instructions. The relative gene expression fold changes were calculated using the $2^{-\Delta \Delta \mathrm{Cq}}$ method (32). The primers used for RT-qPCR in the present study are presented in Table SI.

MPO activity assay. MPO is a marker of activation of neutrophils, and changes in its activity represent the function and activity status of neutrophils. MPO is a clinical examination index for inflammatory bowel disease. In the present study, the measurement of MPO activity in the colon in the homogenates was evaluated using an MPO kit (Nanjing Jiancheng Technology Co., Ltd.) according to the instructions of the manufacturer.

Statistical analyses. The experimental data are expressed as the means \pm standard deviation (SD). Multiple groups were compared by one-way analysis of variance (ANOVA), followed by a Tukey's post hoc test. Data were analyzed using GraphPad software (GraphPad Prism Software, Inc.). Differences were considered statistically significant at P-values $<0.05$.

\section{Results}

Characterization of EVs. EVs were isolated from the supernatant of hP-MSC culture by sequential ultracentrifugation.
The morphology of the EVs was observed by TEM. As shown in Fig. 1A, the EVs exhibited a 'saucer'-like or hemispherical shape with a depression on one side. The results of dynamic light scattering analysis revealed that the diameter of the EVs was approximately $110 \mathrm{~nm}$ (Fig. 1B). The results of western blot analysis indicated that the specific marker of EVs, including CD9, CD63 and Alix were normally expressed and that they were negative for GM130 protein expression (Fig. 1C-F). These results confirmed that the EVs are successfully isolated from the hP-MSCs.

EVs ameliorate TNBS-induced colitis in mice. Following the establishment of the model, the survival, weight loss, colon length, disease activity index (DAI) were observed, and a histological evaluation of the mice with TNBS-induced colitis was performed. The results revealed that the administration of hP-MSC-derived EVs significantly improved clinical parameters, such as the survival rate of the mice with colitis, changes in body weight and DAI compared to the mice treated with PBS (Fig. 2A-C). On the 3rd day following administration, the mice were sacrificed and the colon were collected. As shown in Fig. 2D and E, the length of the colon in the EV group was longer than that in the PBS group. The colon segments of the mice in each group were stained with $H \& E$ to observe the pathological changes. For the histological examination, the results revealed that compared with the PBS group, inflammatory cell infiltration significantly decreased, the number of intestinal glands markedly increased, and crypt abscesses were reduced in the EV-treated group (Fig. 2G). Grading was performed in a blinded manner by a pathologist and the scoring result is presented in Fig. 2F. On the whole, the administration of hP-MSC-derived EVs markedly relieved the symptoms of TNBS-induced IBD in mice. To examine the biodistribution of EVs in the injured colon tissues of mice with colitis, EVs were labeled with Dil (Fig. S1A) to monitor the retention of transplanted EVs by the live animal imaging system. The data revealed the strong red fluorescence signals from the colon tissues at days 0 and 1 following the injection of the Dil-EVs. In comparison with the obtained signals on days 0 and 1 , the fluorescence signals on day 2 were weak. There was almost no fluorescence observed on day 3 (Fig. S1B). 
A

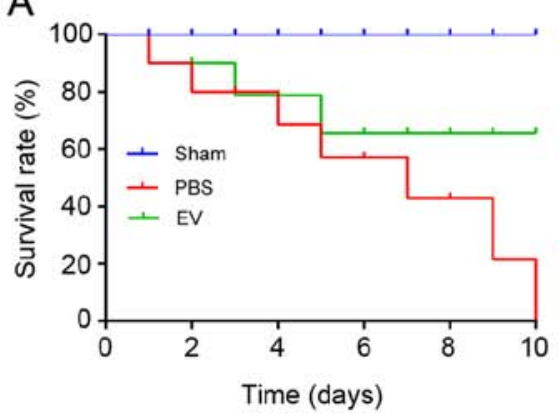

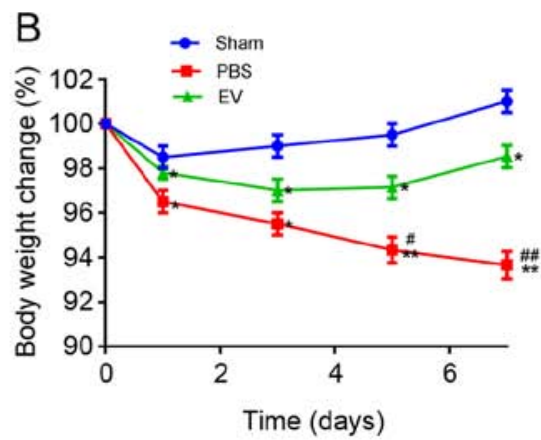

$\mathrm{F}$

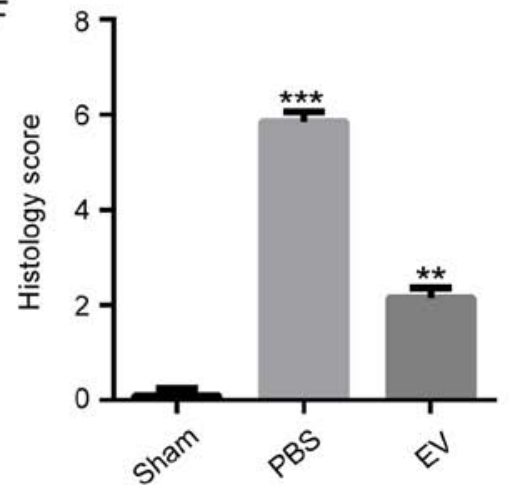

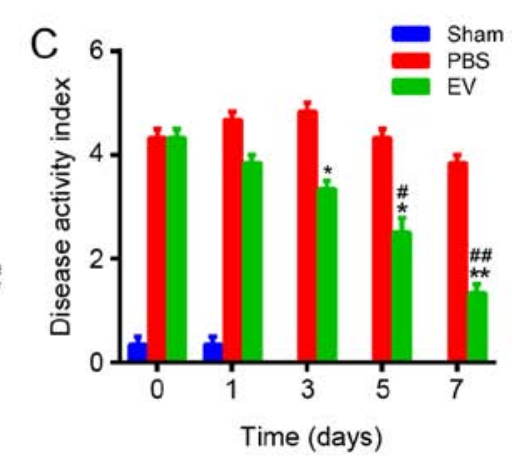

$\mathrm{D}$

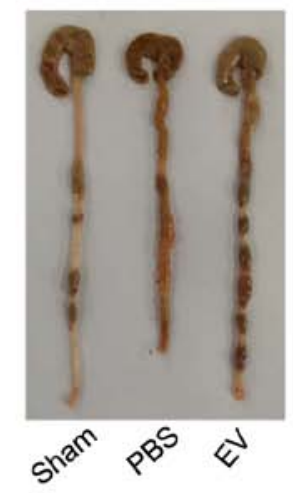

$\mathrm{E}$

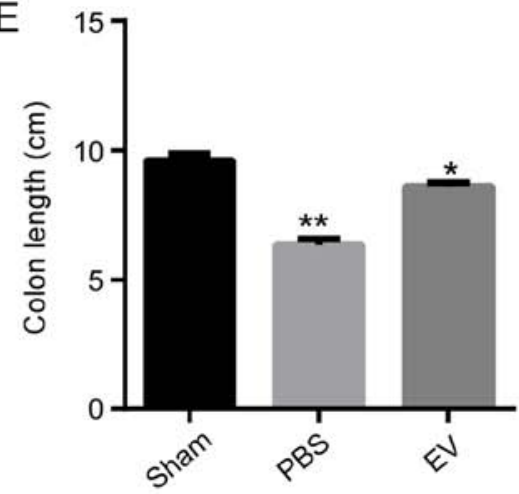

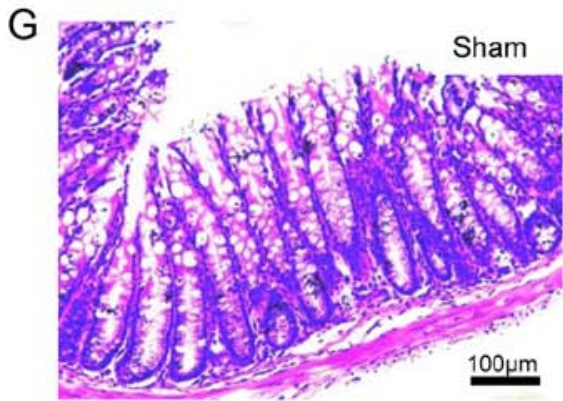
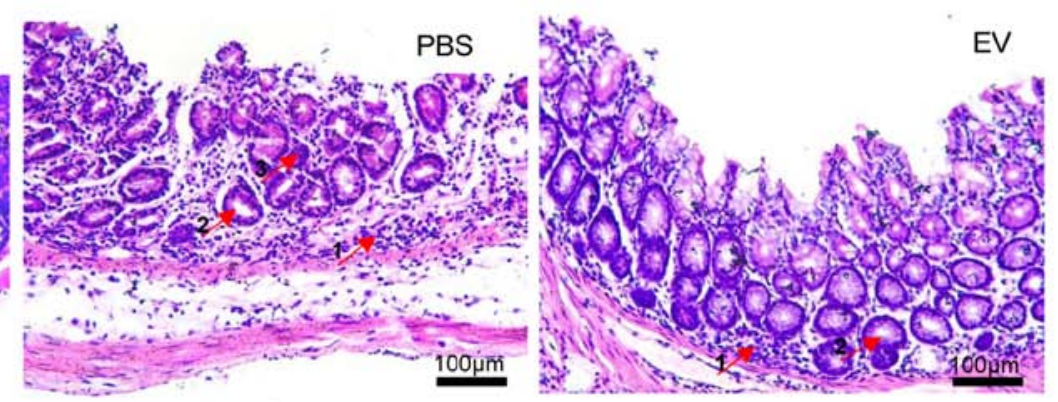

Figure 2. hP-MSC-derived EVs attenuate colitis in mice. (A) Survival rate of mice in the sham, PBS and EV groups (n=8). (B) Percentage of body weight loss following treatment. (C) The disease activity index (DAI) score was used to estimate the severity of symptoms of mice with TNBS induced colitis, seven days in a row (n=8). (D) Macroscopic images of colonic tissues at day 3 after treatment. (E) Colon length in the sham, PBS and EV groups. (F) Histopathological score in the sham, PBS and EV groups. (G) H\&E staining of representative histological sections of mouse colons in the sham, PBS and EV groups. Scale bar, $100 \mu \mathrm{m}$. In the images, the numbered arrows indicate the following: 1 , Inflammatory cells; glands; and 3, crypt abscesses. Data are expressed as the means \pm SD; ( $=8$ ). ${ }^{*} \mathrm{P}<0.05,{ }^{* *} \mathrm{P}<0.01,{ }^{* * *} \mathrm{P}<0.001$ vs. sham group; ${ }^{\#} \mathrm{P}<0.05,{ }^{\# \#} \mathrm{P}<0.01$ vs. PBS group. EVs, extracellular vesicles; hP-MSCs, human placenta-derived mesenchymal stem cells.

EVs reduce intestinal inflammation in mice. To further investigate the mechanisms responsible for the alleviation of the symptoms of colonic inflammation in mice by EVs, the expression of pro-inflammatory and anti-inflammatory cytokines in mouse colon tissues at the gene and protein level were examined by RT-qPCR and western blot analysis, respectively. The results demonstrated that the gene expression levels of pro-inflammatory cytokines (IL-1 $\beta$, TNF- $\alpha$, IL-6 and IFN- $\gamma$ ) in the EV-treated group were significantly lower than those in the PBS group (Fig. 3A). To further corroborate these results, the protein expression levels of pro-inflammatory cytokines were detected by western blot analysis and the amount of target protein was calculated by gray scanning (Figs. 3B and S2). Subsequently, the expression of anti-inflammatory cytokines in the colon tissues of the mice in each group were examined. The results revealed that the expression of anti-inflammatory cytokines (IL-10 and TGF- $\beta$ ) in the EV-treated group were significantly higher than those in the PBS group (Fig. 3C). The results of western blot analysis revealed that the levels of anti-inflammatory factors (IL-10 and TGF- $\beta$ ) increased significantly in the EV-treated group compared with the PBS group (Fig. 3D). These results indicated that the hP-MSC-derived EVs attenuated colitis by regulating the balance between pro-inflammatory and anti-inflammatory cytokines in mice with TNBS-induced colitis.

Effect of EV administration on antioxidant defenses in the colitis mice. To examine the therapeutic effects of hP-MSC-derived EVs on oxidative stress in TNBS-induced colitis, the IVIS Lumina Imaging System was used to measure the levels of ROS in colon tissues. EV administration significantly decreased the ROS content, and the light signal on the 7 th day was extremely low, indicating a lower ROS content and a reduced inflammatory response (Fig. 4A and B). Moreover, 

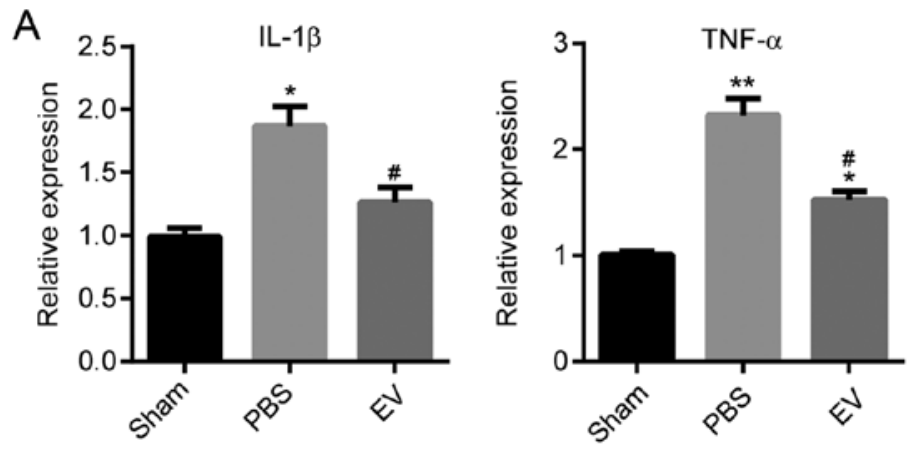

B
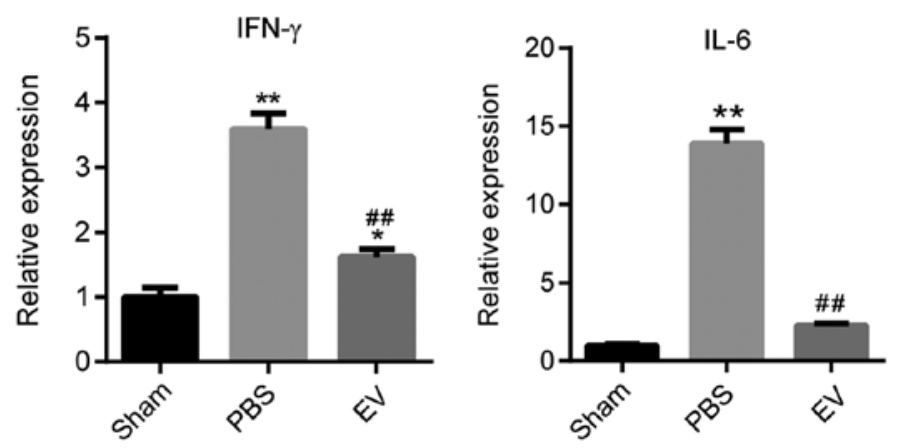

IL-1 $\beta$

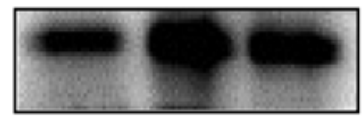

TNF- $\alpha$

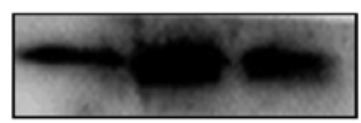

IFN-y

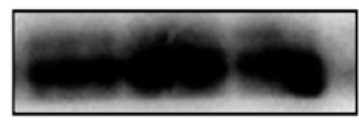

IL-6

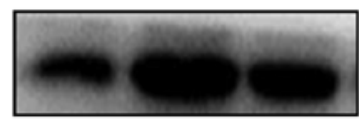

$\beta$-actin

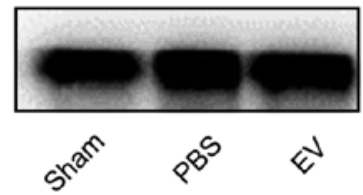

C
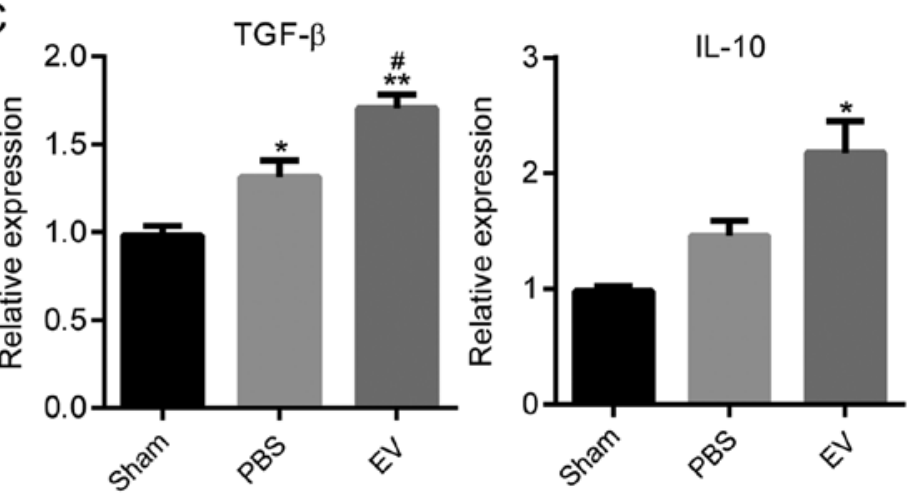

TGF- $\beta$

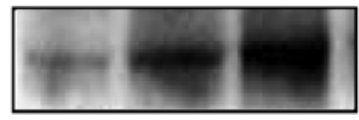

IL-10

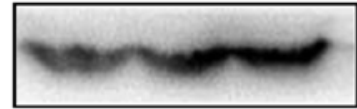

$\beta$-actin

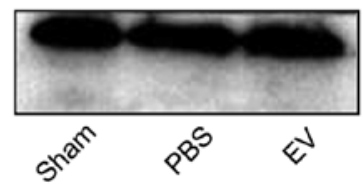

Figure 3. Expression levels of pro-inflammatory and anti-inflammatory factors in colon tissues. (A) The expression of pro-inflammatory factors (IL-1 $\beta$, IL-6, TNF- $\alpha$ and IFN- $\gamma$ ) was measured by using RT-qPCR. (B) The protein levels of pro-inflammatory factors were measured by using western blot analysis. (C) The expression of anti-inflammatory factors (IL-10, TGF- $\beta$ ) was measured by using RT-qPCR. (D) The protein level of anti-inflammatory factor was measured by using western blot analysis. Data are expressed as the means $\pm \mathrm{SD}$. ${ }^{*} \mathrm{P}<0.05,{ }^{* *} \mathrm{P}<0.01$ vs. sham group; ${ }^{\#} \mathrm{P}<0.05$, ${ }^{\# \#} \mathrm{P}<0.01$ vs. $\mathrm{PBS}$ group. EVs, extracellular vesicles.

MPO activity was measured in the colon homogenate to determine the number of activated polymorphonuclear leukocytes. The results revealed that MPO activity was significantly downregulated in the EV-treated group compared with the PBS group (Fig. 4C). The above-mentioned results were further confirmed by immunohistochemical staining for MPO (Figs. 4D and S3). These results suggested that EVs inhibited the oxidative stress reaction in the mice with colitis.

EVs downregulate the expression of apoptotic proteins. The expression of caspase- 3 , caspase- 8 and caspase- 9 at the gene level was determined, as markers of colonic cell apoptosis. In comparison to the PBS group, the levels of apoptosis-related genes were markedly decreased in the mice with colitis administered EVs (Fig. 5A). The results of the analysis based on the immumohistochemical staining of caspase-3 protein expression were in accordance with those of RT-qPCR (Fig. 5B). The mean of integrated option density (IOD) of caspase-3 was measured with imaging analysis and then statistically analyzed (Fig. S4). All these data indicated that the administration of EVs played an important role in protecting epithelial cells from injury in mice with colitis.

EVs promote mucosal healing in mice with colitis. The present study then investigated whether EVs play a role in intestinal epithelial cells (IECs) in mice with colitis. The assessment of Cldn1, ZO-1 and Occludin mRNA expression by RT-qPCR revealed that their expression markedly increased in the $\mathrm{EV}$ group compared with the PBS group (Fig. 6A). In contrast to the above-mentioned results, the mRNA expression levels of MMP-2 and MMP-9 were decreased in EV group (Fig. 6B). Subsequently, the expression of EpCAM was examined in the different groups. The results of western blot analysis revealed that the expression of EpCAM in EVs group were significantly higher than that in the PBS group (Fig. 6C). In addition, EpCAM immunofluorescence staining and immunohistochemical staining revealed that the colonic tissues from the mice in the 

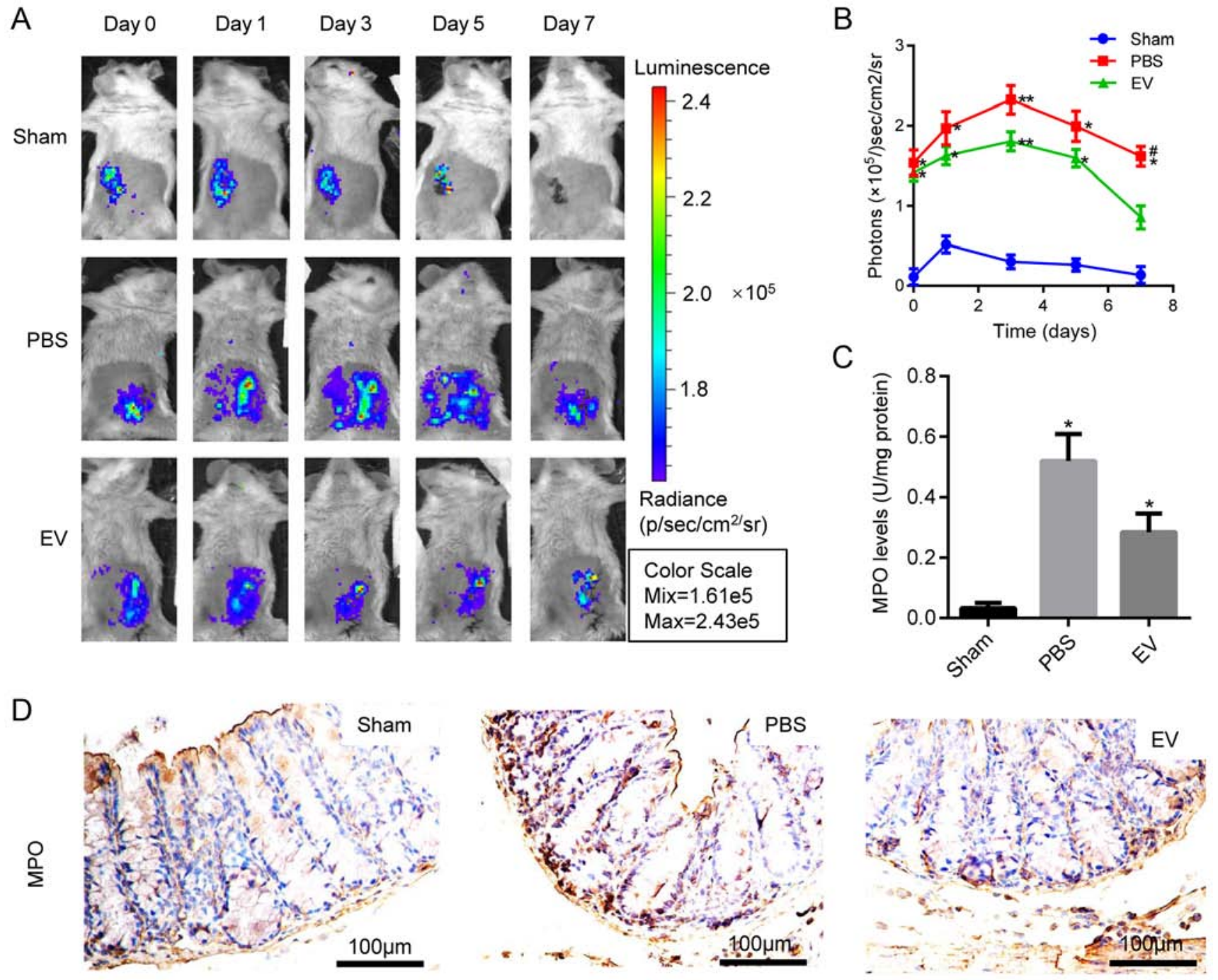

Figure 4. Detection of indexes about oxidative stress. (A) ROS activities in mice with colitis tracked by bioluminescence imaging (BLI) in vivo. (B) Quantitative analysis of BLI signals intensity $(\mathrm{n}=8)$. (C) Myeloperoxidase assay by using MPO detection kits. The supernatant collected from colon homogenate assayed by spectrophotometry for MPO activity determination at $450 \mathrm{~nm}$. ${ }^{*} \mathrm{P}<0.05,{ }^{* *} \mathrm{P}<0.01$ vs. sham group; ${ }^{*} \mathrm{P}<0.05$ vs. PBS group. (D) Representative photographs of immunohistochemical staining of MPO in the colon tissues of mice in Sham, PBS, and EVs groups. (n=8). Scale bar, 100 $\mu \mathrm{m}$. The specific positive coloration of MPO is shown in brown color. EVs, extracellular vesicles.

EV group maintained a better epithelial integrity, compared with those from mice in the PBS group (Fig. S5A and B). The immunohistochemical-mediated examination of $\mathrm{Ki} 67$ protein expression revealed that the EVs promoted the proliferation of IECs (Figs. 6D and S6).

\section{Discussion}

In the present study, EVs derived from hP-MSCs were utilized for the treatment of colitis by in situ injection. The results revealed that the $\mathrm{hP}-\mathrm{MSC}$-derived EVs attenuated TNBS-induced colitis, as assessed by body weight loss and the DAI histological scores. Furthermore, it was found that the administration of hP-MSC-derived EVs markedly reduced intestinal inflammation and oxidative stress.

A number of studies have demonstrated that hP-MSC-based therapy could be developed as a potential treatment for several diseases $(10,33,34)$. The application of hP-MSCs in various disease models has several advantages (34). First, the placenta has wide resources and the use of this is not associated with any ethical concerns. Second, hP-MSCs are easily available and have a low immunogenicity and viral infection rate, which have more widespread application prospect than bone marrow-MSCs. Moreover, MSCs derived from umbilical cord blood have unavoidable immunogenicity, and the available amniotic fluid is limited. Therefore, hP-MSCs are a better choice, compared with other sources. In addition, a previous study demonstrated that hP-MSCs retained a higher therapeutic efficacy than bone marrow-derived MSCs in a model of hindlimb ischemia disease (35). MSC-derived EVs reflect the characteristics of their source cells; thus, there are differences between different stem cell-derived EVs. There are marked differences in growth factors and cytokines secreted by MSCs from different sources. Studies have reported that EVs derived from MSCs mainly exert biological effects via the following mechanisms $(16,20,36,37)$ : Surface protein molecules and lipid ligands of EVs can directly bind to related receptors on the surface of target cells and further activate the signaling pathways in target cells; EVs can directly enter target cells through fusion or endocytosis with the target cell, so that the 
A

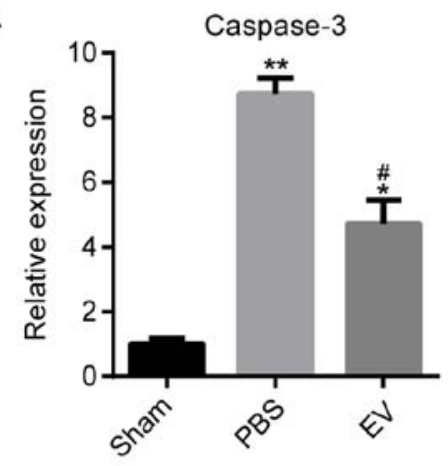

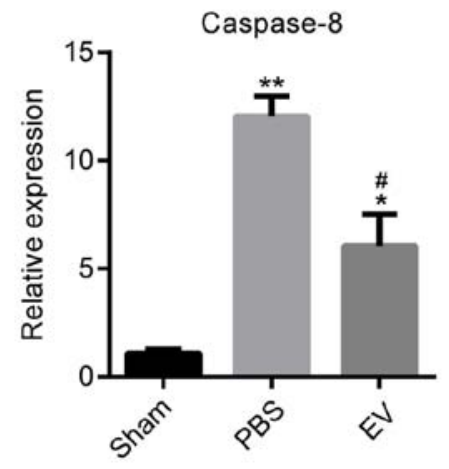

B
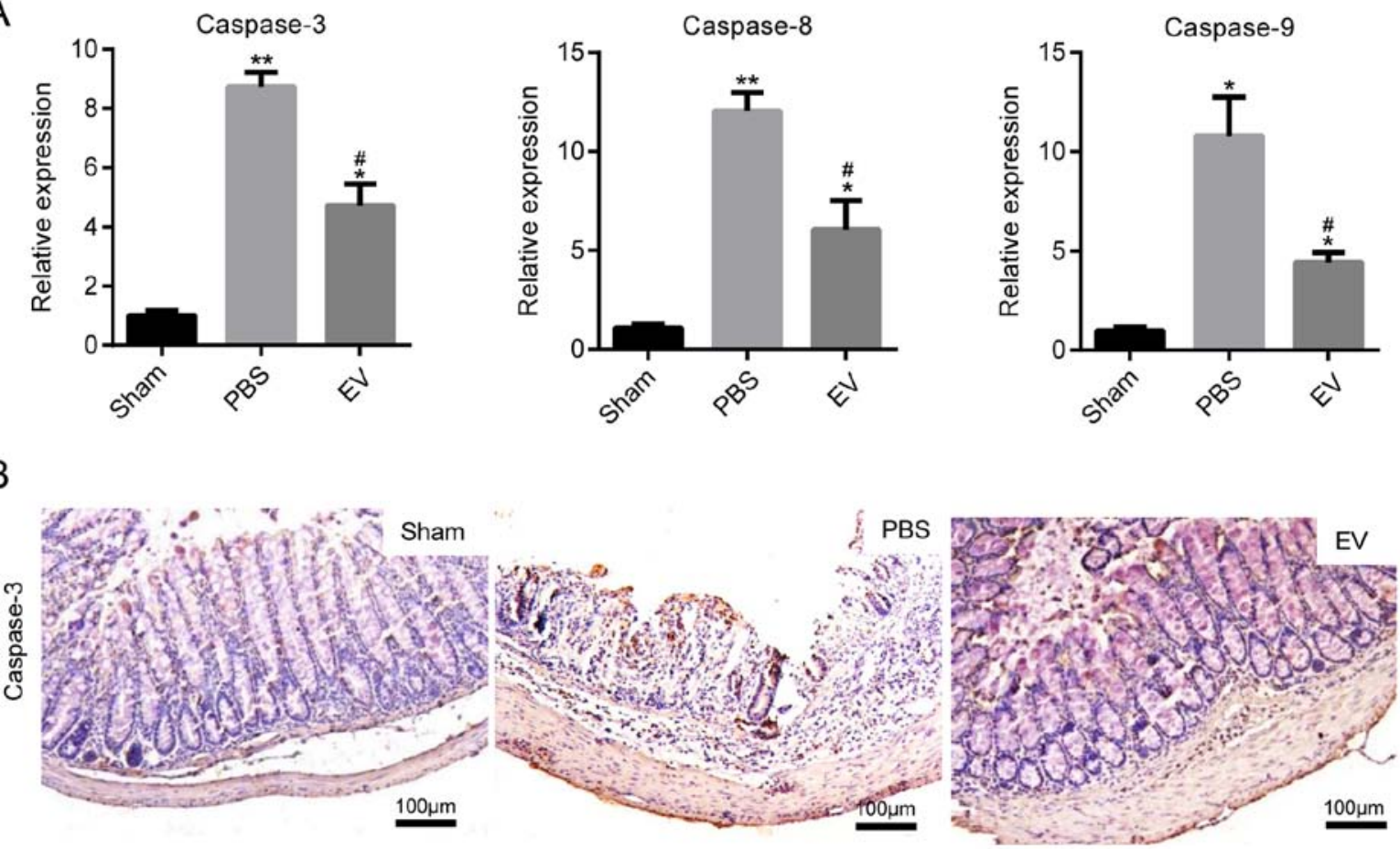

Figure 5. EVs downregulate the expression of apoptotic proteins. (A) The expression of apoptosis-related genes (caspase-3, caspase-8 and caspase-9) were measured by RT-qPCR. Scale bar, $100 \mu \mathrm{m}$. Data are expressed as the means \pm SD. ${ }^{*} \mathrm{P}<0.05,{ }^{* *} \mathrm{P}<0.01$ vs. sham group; ${ }^{*} \mathrm{P}<0.05$ vs. PBS group. (B) Representative images of immunohistochemical staining of caspase- 3 in the colon tissues of mice in the sham, PBS and EV groups; (n=8). Scale bar, $100 \mu \mathrm{m}$. The specific positive coloration of caspase- 3 is shown in brown color. EVs, extracellular vesicles.

bioactive substances such as proteins, lipid and nucleic acid carried by them can be brought into the target cell, to further regulate the function of the target cell and exert the therapeutic effect. EVs derived from MSCs carry a variety of biologically active substances (18). The exact molecular mechanisms of action EVs remain unknown, and in future studies, the authors aim to investigate and clarify the types of components in EVs which play therapeutic roles.

Cytokine responses play an important role in the pathophysiology of IBD, including Crohn's disease and ulcerative colitis (38). Furthermore, the cytokine responses of IBD continue during the entire process of inflammation, and are also a major pathophysiological factor in the eventual resolution of inflammation (39). In particular, intestinal inflammation and the destruction of the intestinal mucosa are related to the imbalance in pro-inflammatory and anti-inflammatory cytokines (40). Therefore, the development of strategies with which to regulate this imbalance would be an effective treatment for IBD. In the present study, it was confirmed that the administration of EVs downregulated the expression of pro-inflammatory cytokines (TNF- $\alpha$, IL- $1 \beta$, IFN- $\gamma$ and IL-6) in colon tissues of mice with colitis, compared to those treated with PBS. On the contrary, the administration of EVs upregulated the expression of anti-inflammatory cytokines, such as IL-10 and TGF- $\beta$. These results indicated that EV administration regulated the balance of pro-inflammatory and anti-inflammatory cytokines in mice with TNBS-induced colitis.

A growing body of evidence has indicated that oxidative stress plays a crucial role in the pathogenesis and progression of IBD, and is considered to be involved in the infiltration and activation of neutrophils in damaged intestinal tissue $(41,42)$.
The increase in ROS generation and the decrease in antioxidant activities could contribute to the major pathogenesis of IBD (43). MPO, as a biochemical indicator of neutrophil infiltration and activation in the intestinal mucosa, is widely applied to evaluate the severity of intestinal inflammation in IBD (44). MPO secreted by the activated neutrophils, a potent oxidant, plays an important role in oxidative stress by catalyzing hydrogen dioxide $\left(\mathrm{H}_{2} \mathrm{O}_{2}\right)$ to hypochlorite (HOCL) (41). The present study demonstrated that the administration of hP-MSC-derived EVs markedly decreased the accumulation of neutrophils and MPO activity in injured sites in mice with colitis. The dynamic equilibrium of ROS production and metabolism is crucial for the maintenance of the normal function of cells and tissues, if this balance is disrupted, it can lead to oxidative stress and a series of tissue damage (43). The results of the present study further confirmed that the hP-MSC-derived EVs decreased the levels of ROS in mice with TNBS-induced colitis and protected the cells against oxidative damage.

Furthermore, the formation of intestinal damage in IBD is closely related to the production of ROS, and the overload of ROS alter tight junctions and lead to pathological changes in epithelial permeability. Previous studies have found that oxidative stress may cause apoptosis. Abnormal apoptosis may lead to the disruption of intestinal mucosal integrity, and induce the invasion of pathogenic agents and bacteria, resulting in the continuous activation of intestinal T-cells, and pro-inflammatory cytokines can further promote IEC apoptosis in this process $(43,45)$. In order to investigate the disorder of colonic epithelium apoptosis in mice with TNBS-induced colitis, the present study detected the expression of certain genes and 
A

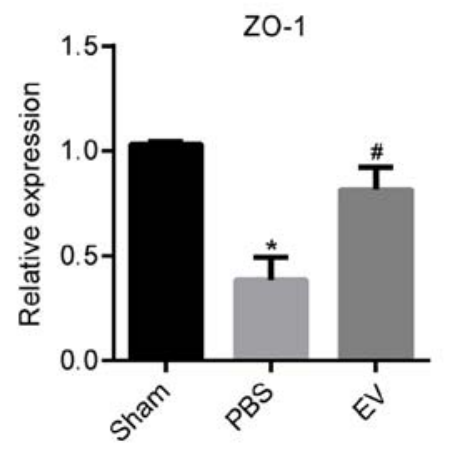

B

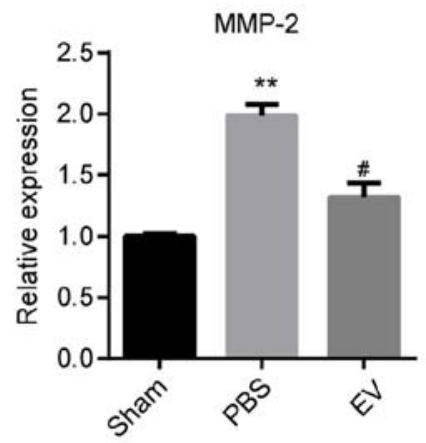

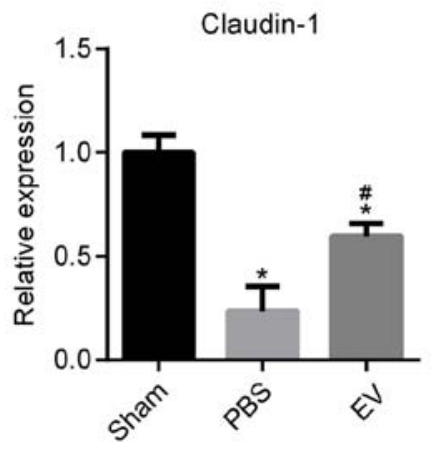

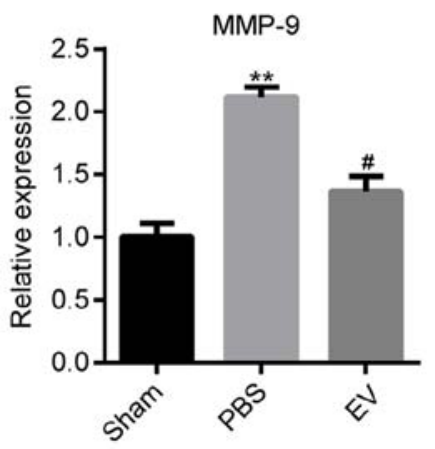

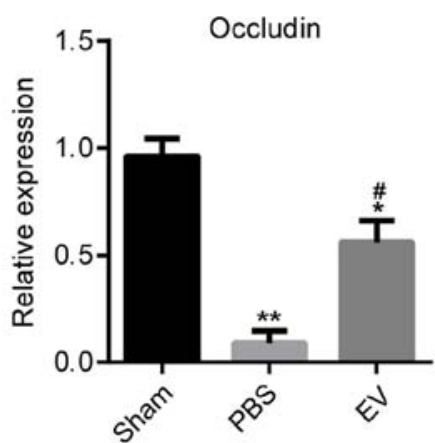

C

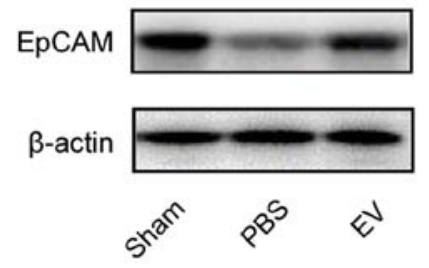

D

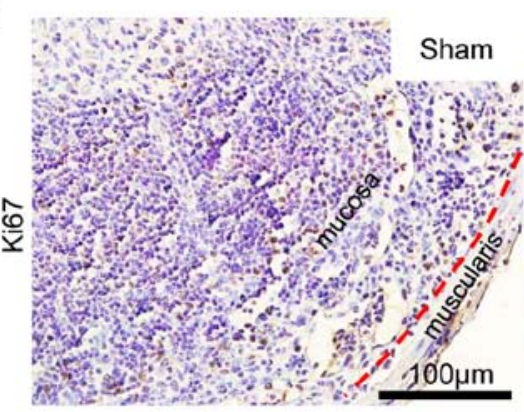

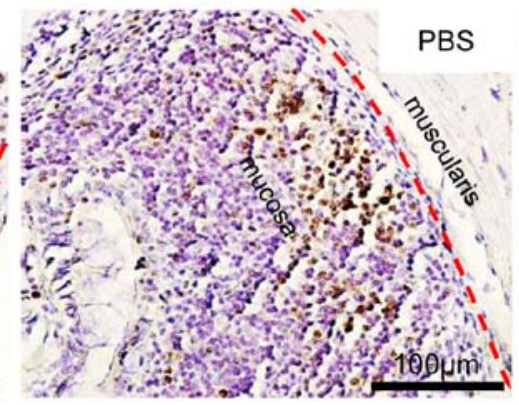

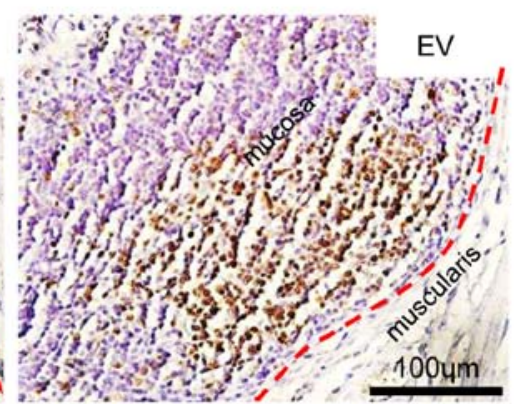

Figure 6. Promoting the proliferation of IECs and maintaining tight junctions. (A) The expression of tight junction proteins (Claudin-1, ZO-1 and Occludin) was measured by RT-qPCR. (B) The expression of matrix metalloproteinase (MMP-2 and MMP-9) was measured by using RT-qPCR. Data are expressed as the means $\pm \mathrm{SD}$. ${ }^{*} \mathrm{P}<0.05,{ }^{* *} \mathrm{P}<0.01$ vs. sham group; ${ }^{*} \mathrm{P}<0.05$ vs. PBS group. (C) The protein level of EpCAM was measured by western blot analysis (D) Representative images of immunohistochemical staining of Ki67 in the colon tissues of mice in sham, PBS and EV groups; (n=8). Scale bar, $100 \mu \mathrm{m}$. The specific positive coloration of Ki67 is shown in brown. EVs, extracellular vesicles.

protein related to apoptosis. Following the administration of hP-MSC-derived EVs, it was found that the EVs decreased the gene expression levels of caspase-3, caspase-8 and caspase- 9 , and decreased the protein expression of caspase-3.

Mucosal healing has been regarded as an important therapeutic goal in IBD, and the evaluation of mucosal healing is based on the integrity of the gut epithelium. The integrity of the epithelial monolayer is formed by IECs with intercellular junctions between adjacent cells (46), which creates the continuous physical barrier (47). The gastric mucosal barrier is the first barrier to come in contact with the intestinal tract and external environment, and plays an important role in reducing the invasion of pathogens and the absorption of toxins. Although the intestinal tract is a protective barrier, pathogens or other substances in the intestinal tract can stimulate IECs and polymorphonuclear neutrophils to secrete inflammatory mediators and promote oxidative stress. When damaged, it can lead to an increase in intestinal epithelial permeability, which plays an important role in the pathogenesis of IBD. In order to maintain the integrity of the gut epithelium and the intestinal barrier, IECs need to proliferate constantly, which is crucial for mucosal healing $(48,49)$. Tight junction proteins, such as Cldn1, Zo1 and Occludin, function as indicators for the evaluation of colonic epithelial integrity in mice with colitis (50). In the present study, it was found that hP-MSC-derived EVs maintained proper tight junctions in the colons of the treated mice, and promoted the proliferation of the colon epithelial cells. Taken together, these results indicated that hP-MSC-derived EVs played an important role in promoting mucosal healing.

In conclusion, the present study developed a method for treatment of experimental colitis in mice with the use of hP-MSC-derived EVs. EVs markedly relieved the clinical symptoms by inhibiting inflammation and oxidative stress to promote mucosal healing in mice with TNBS-induced colitis. Moreover, molecular imaging used in the present study elucidated the effects of EV administration on antioxidant defenses in mice with colitis by the real-time tracking of ROS in living mice. In general, the findings of the present study provide new insight into the treatment of IBD. EVs derived from hP-MSCs may be used as a novel therapeutic strategy for IBD. 


\section{Acknowledgements}

Not applicable.

\section{Funding}

The present study was partially supported by granst from the National Key R\&D Program of China (no. 2017YFA0103200), National Natural Science Foundation of China (no. 81671734), Key Projects of Tianjin Science and Technology Support Program (no. 18YFZCSY00010), and National Natural Science Foundation of China (no. 81470808).

\section{Availability of data and materials}

All data generated and/or analyzed during this study are available from the corresponding author upon reasonable request.

\section{Authors' contributions}

ZL, ZG and XC conceived and designed the experiments. LD performed the majority of the experiments. $\mathrm{HH}, \mathrm{XZ}, \mathrm{MZ}, \mathrm{SC}$, $\mathrm{CW}, \mathrm{ZH}$ and $\mathrm{ZCH}$ collected and analyzed the data. LD and ZL wrote the main manuscript and prepared the figures. ZL provided funding. All authors have read and approved the final manuscript.

\section{Ethics approval and consent to participate}

The protocols involved animals and the experimental procedures of the present study were approved by the Nankai University Animal Care and Use Committee guidelines (Approval no. 20170022) and conducted according to the international regulations of the usage and welfare of laboratory animals. All experimental procedures were conducted in accordance with the Tianjin Committee for the Use and Care of Laboratory Animals. All research was conducted in accordance with the provided protocol. The animal experiment strictly adhered to the principles of using the least number of animals to complete the experiment and minimizing the pain of the experimental animals.

\section{Patient consent for publication}

Not applicable.

\section{Competing interests}

The authors declare that they have no competing interests.

\section{References}

1. Kim DH and Cheon JH: Pathogenesis of inflammatory bowel disease and recent advances in biologic therapies. Immune Netw 17: 25-40, 2017

2. Chen X, Cai C, Xu D, Liu Q, Zheng S, Liu L, Li G, Zhang X, Li X, Ma Y, et al: Human mesenchymal stem cell-treated regulatory $\mathrm{CD} 23+\mathrm{CD} 43+\mathrm{B}$ cells alleviate intestinal inflammation. Theranostics 9: 4633-4647, 2019.

3. Manichanh C, Borruel N, Casellas F and Guarner F: The gut microbiota in IBD. Nat Rev Gastroenterol Hepatol 9: 599-608, 2012.
4. Baumgart DC and Sandborn WJ: Crohn's disease. Lancet 380: 1590-1605, 2012.

5. Ordás I,Eckmann L, Talamini M, Baumgart DC and Sandborn WJ: Ulcerative colitis. Lancet 380: 1606-1619, 2012.

6. Chudy-Onwugaje KO, Christian KE, Farraye FA and Cross RK: A state-of-the-art review of new and emerging therapies for the treatment of IBD. Inflamm Bowel Dis 25: 820-830, 2019.

7. Dave M, Mehta K, Luther J, Baruah A, Dietz AB and Faubion WA Jr: Mesenchymal stem cell therapy for inflammatory bowel disease: A systematic review and meta-analysis. Inflamm Bowel Dis 21: 2696-2707, 2015.

8. González MA, Gonzalez-Rey E, Rico L, Büscher D and Delgado M: Adipose-derived mesenchymal stem cells alleviate experimental colitis by inhibiting inflammatory and autoimmune responses. Gastroenterology 136: 978-989, 2009.

9. Ricart E: Current status of mesenchymal stem cell therapy and bone marrow transplantation in IBD. Dig Dis 30: 387-391, 2012.

10. Tao H, Han Z, Han ZC and Li Z: Proangiogenic features of mesenchymal stem cells and their therapeutic applications. Stem Cells Int 2016: 1314709, 2016

11. Cosenza S, ToupetK, Maumus M,Luz-Crawford P,Blanc-Brude O, Jorgensen C and Noël D: Mesenchymal stem cells-derived exosomes are more immunosuppressive than microparticles in inflammatory arthritis. Theranostics 8: 1399-1410, 2018.

12. Liu Y, Cui J, Wang H, Hezam K, Zhao X, Huang H, Chen S, Han Z, Han ZC, Guo Z and Li Z: Enhanced therapeutic effects of MSC-derived extracellular vesicles with an injectable collagen matrix for experimental acute kidney injury treatment. Stem Cell Res Ther 11: 161, 2020.

13. Zhang K, Zhao X, Chen X, Wei Y, Du W, Wang Y, Liu L, Zhao W, Han Z, Kong D, et al: Enhanced therapeutic effects of mesenchymal stem cell-derived exosomes with an injectable hydrogel for hindlimb ischemia treatment. ACS Appl Mater Interfaces 10: 30081-30091, 2018.

14. Qi X, Zhang J, Yuan H, Xu Z, Li Q, Niu X, Hu B, Wang Y and Li X: Exosomes secreted by human-induced pluripotent stem cell-derived mesenchymal stem cells repair critical-sized bone defects through enhanced angiogenesis and osteogenesis in osteoporotic rats. Int J Biol Sci 12: 836-849, 2016.

15. Tao H, Chen X, Cao H, Zheng L, Li Q, Zhang K, Han Z, Han ZC, Guo Z, Li Z and Wang L: Mesenchymal stem cell-derived extracellular vesicles for corneal wound repair. Stem Cells Int 2019: $5738510,2019$.

16. Tkach $\mathrm{M}$ and Théry $\mathrm{C}$ : Communication by extracellular vesicles: Where we are and where we need to go. Cell 164: 1226-1232, 2016.

17. Bei Y, Das S, Rodosthenous RS, Holvoet P, Vanhaverbeke M, Monteiro MC, Monteiro VVS, Radosinska J, Bartekova M, Jansen F, et al: Extracellular vesicles in cardiovascular theranostics. Theranostics 7: 4168-4182, 2017.

18. Du W, Zhang K, Zhang S, Wang R, Nie Y, Tao H, Han Z, Liang L, Wang D, Liu J, et al: Enhanced proangiogenic potential of mesenchymal stem cell-derived exosomes stimulated by a nitric oxide releasing polymer. Biomaterials 133: 70-81, 2017.

19. Yu B, Zhang X and Li X: Exosomes derived from mesenchymal stem cells. Int J Mol Sci 15: 4142-4157, 2014.

20. Qiu G, Zheng G, Ge M, Wang J, Huang R, Shu Q and Xu J: Mesenchymal stem cell-derived extracellular vesicles affect disease outcomes via transfer of microRNAs. Stem Cell Res Ther 9: 320, 2018.

21. Liao Z, Luo R, Li G, Song Y, Zhan S, Zhao K, Hua W, Zhang Y, $\mathrm{Wu} \mathrm{X}$ and Yang C: Exosomes from mesenchymal stem cells modulate endoplasmic reticulum stress to protect against nucleus pulposus cell death and ameliorate intervertebral disc degeneration in vivo. Theranostics 9: 4084-4100, 2019.

22. Zhang Y, Xu J, Liu S, Lim M, Zhao S, Cui K, Zhang K, Wang L, Ji Q, Han Z, et al: Embryonic stem cell-derived extracellular vesicles enhance the therapeutic effect of mesenchymal stem cells. Theranostics 9: 6976-6990, 2019.

23. Rani S, Ryan AE, Griffin MD and Ritter T: Mesenchymal stem cell-derived extracellular vesicles: Toward cell-free therapeutic applications. Mol Ther 23: 812-823, 2015.

24. Tan SS, Yin Y, Lee T, Lai RC, Yeo RW, Zhang B, Choo A and Lim SK: Therapeutic MSC exosomes are derived from lipid raft microdomains in the plasma membrane. J Extracell Vesicles 2, 2013.

25. Anderson JD, Johansson HJ, Graham CS, Vesterlund M, Pham MT, Bramlett CS, Montgomery EN, Mellema MS, Bardini RL, Contreras Z, et al: Comprehensive proteomic analysis of mesenchymal stem cell exosomes reveals modulation of angiogenesis via nuclear factor-kappaB signaling. Stem Cells 34: 601-613, 2016. 
26. Mao F, Wu Y, Tang X, Kang J,Zhang B, Yan Y, Qian H, Zhang X and $\mathrm{Xu}$ W: Exosomes derived from human umbilical cord mesenchymal stem cells relieve inflammatory bowel disease in mice. Biomed Res Int 2017: 5356760, 2017.

27. Hoshino A, Costa-Silva B, Shen TL, Rodrigues G, Hashimoto A, Tesic Mark M, Molina H, Kohsaka S, Di Giannatale A, Ceder S, et al: Tumour exosome integrins determine organotropic metastasis. Nature 527: 329-335, 2015.

28. Chen G, Ran X, Li B, Li Y, He D, Huang B, Fu S, Liu J and Wang W: Sodium butyrate inhibits inflammation and maintains epithelium barrier integrity in a TNBS-induced inflammatory bowel disease mice model. EBioMedicine 30: 317-325, 2018.

29. Zhang S, Liu Y, Zhang X, Zhu D, Qi X, Cao X, Fang Y, Che Y, Han ZC, He ZX, et al: Prostaglandin $\mathrm{E}_{2}$ hydrogel improves cutaneous wound healing via M2 macrophages polarization. Theranostics 8: 5348-5361, 2018.

30. Zhang K, Wang C, Wang R, Chen S and Li Z: Dual bioluminescence imaging of tumor progression and angiogenesis. J Vis Exp: e59763, 2019

31. Zhao N, Yue Z, Cui J, Yao Y, Song X, Cui B, Qi X, Han Z, Han ZC, Guo Z, et al: IGF-1C domain-modified hydrogel enhances therapeutic potential of mesenchymal stem cells for hindlimb ischemia. Stem Cell Res Ther 10: 129, 2019.

32. Livak KJ and Schmittgen TD: Analysis of relative gene expression data using real-time quantitative PCR and the 2(-Delta Delta C(T)) method. Methods 25: 402-408, 2001.

33. Liang L, Li Z, Ma T, Han Z, Du W, Geng J, Jia H, Zhao M, Wang J, Zhang B, et al: Transplantation of human placenta-derived mesenchymal stem cells alleviates critical limb ischemia in diabetic nude rats. Cell Transplant 26: 45-61, 2017.

34. Li Z and Han ZC: Introduction of perinatal tissue-derived stem cells. In: Perinatal Stem Cells: Biology, Manufacturing and Translational Medicine. Han ZC, Takahashi TA, Han Z and Li Z (eds). Springer Singapore, Singapore, pp1-7, 2019.

35. Jeon YJ, Kim J, Cho JH, Chung HM and Chae JI: Comparative analysis of human mesenchymal stem cells derived from bone marrow, placenta, and adipose tissue as sources of cell therapy. J Cell Biochem 117: 1112-1125, 2016.

36. Keshtkar S, Azarpira N and Ghahremani MH: Mesenchymal stem cell-derived extracellular vesicles: Novel frontiers in regenerative medicine. Stem Cell Res Ther 9: 63, 2018.

37. Hong P, Yang H, Wu Y, Li K and Tang Z: The functions and clinical application potential of exosomes derived from adipose mesenchymal stem cells: A comprehensive review. Stem Cell Res Ther 10: 242, 2019.

38. Neurath MF: Cytokines in inflammatory bowel disease. Nat Rev Immunol 14: 329-342, 2014.

39. Strober W and Fuss IJ: Proinflammatory cytokines in the pathogenesis of inflammatory bowel diseases. Gastroenterology 140: $1756-1767,2011$
40. Guan Q and Zhang J: Recent advances: The imbalance of cytokines in the pathogenesis of inflammatory bowel disease. Mediators Inflamm 2017: 4810258, 2017.

41. Brito TV, Neto JP, Prudêncio RS, Batista JA, Júnior JS, Silva RO, Franco AX, Aragão KS, Soares PM, Souza MH, et al: Sulfated-polysaccharide fraction extracted from red algae gracilaria birdiae ameliorates trinitrobenzenesulfonic acid-induced colitis in rats. J Pharm Pharmacol 66: 1161-1170, 2014.

42. Denson LA, Jurickova I, Karns R, Shaw KA, Cutler DJ, Okou DT, Dodd A, Quinn K, Mondal K, Aronow BJ, et al: Clinical and genomic correlates of neutrophil reactive oxygen species production in pediatric patients with Crohn's disease. Gastroenterology 154: 2097-2110,2018.

43. Tian T, Wang $Z$ and Zhang J: Pathomechanisms of oxidative stress in inflammatory bowel disease and potential antioxidant therapies. Oxid Med Cell Longev 2017: 4535194, 2017.

44. Hansberry DR, Shah K, Agarwal P and Agarwal N: Fecal myeloperoxidase as a biomarker for inflammatory bowel disease. Cureus 9: e1004, 2017

45. Souza HS, Tortori CJ, Castelo-Branco MT, Carvalho AT, Margallo VS, Delgado CF, Dines I and Elia CC: Apoptosis in the intestinal mucosa of patients with inflammatory bowel disease: Evidence of altered expression of FasL and perforin cytotoxic pathways. Int J Colorectal Dis 20: 277-286, 2005.

46. Martini E, Krug SM, Siegmund B, Neurath MF and Becker C: Mend your fences: The epithelial barrier and its relationship with mucosal immunity in inflammatory bowel disease. Cell Mol Gastroenterol Hepatol 4: 33-46, 2017.

47. Peterson LW and Artis D: Intestinal epithelial cells: Regulators of barrier function and immune homeostasis. Nat Rev Immunol 14: 141-153, 2014.

48. Boltin D, Perets TT, Vilkin A and Niv Y: Mucin function in inflammatory bowel disease: An update. J Clin Gastroenterol 47: 106-111, 2013.

49. Johansson ME, Sjövall H and Hansson GC: The gastrointestinal mucus system in health and disease. Nat Rev Gastroenterol Hepatol 10: 352-361, 2013.

50. Marchiando AM, Shen L, Graham WV, Edelblum KL, Duckworth CA, Guan Y, Montrose MH, Turner JR and Watson AJ: The epithelial barrier is maintained by in vivo tight junction expansion during pathologic intestinal epithelial shedding. Gastroenterology 140: 1208-1218.e1-e2, 2011.

This work is licensed under a Creative Commons Attribution-NonCommercial-NoDerivatives 4.0 International (CC BY-NC-ND 4.0) License. 